\title{
ANALYTIC METHOD TO COMPUTE THE ISOSTATICS USING THE ISOCLINIC FRINGES
}

\section{OANTA, E[mil]; PANAIT, C[ornel]; SABAU, A[drian]; BARHALESCU, M[ihaela] L[uminita] \& AXINTE, T[iberiu]}

\begin{abstract}
Research projects require advanced and appropriate means of investigation which might use theoretical approaches like analytic methods, numerical methods as well as experimental studies. A high degree of integration may be achieved if the main instrument to be used is the computer. Moreover, the effectiveness of each study is increased if computer based methods are conceived. From this standpoint, the paper presents an analytic method to compute the points which define the isostatic curves on the surface of the photoelastic coating, using the isoclines defined as sets of points. Several methods to process the data are presented and a computer code may be easily developed using these relations; this computer code may increase the effectiveness of the data processing in terms of accuracy, time and volume of the data.

Keywords:Experimental mechanics, analytic method, computer aided instrument
\end{abstract}

\section{INTRODUCTION}

Research projects require advanced and appropriate means of investigation which might use theoretical approaches like analytic methods, numerical methods as well as experimental studies. At the upmost concept level, one can identify theoretical models, like analytic and numeric models and experimental models, figure 1. Experimental models are paramount for the success of a research because they offer the real data employed either to calibrate the theoretical models, or to check the accuracy of the results.

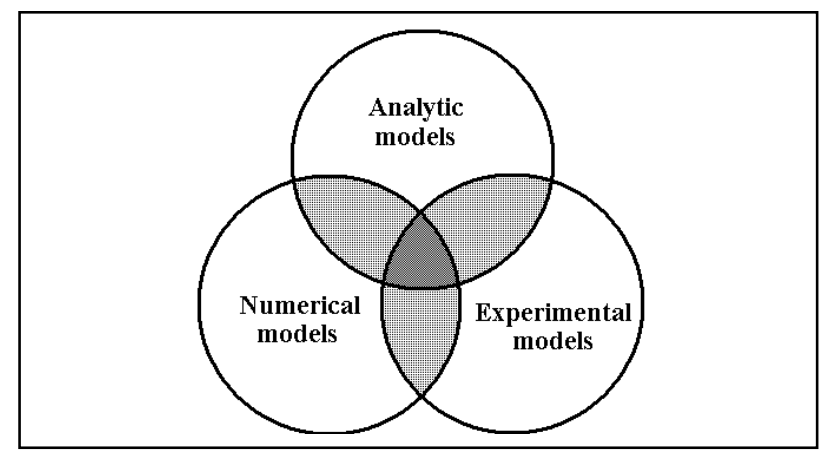

Fig. 1. Methods to acquire knowledge in research

Data integration between these studies can be easily done if an original software is employed. In this way we must remind that hybrid modeling and development of original software for research, design and education is a long run concern of the authors.

From this standpoint, the mathematical background (analytic and numeric) of the original software applications must be conceived in an algorithmic way in order to offer flexibility and reusability of the code, [2], [3], [4], and [5].

Regarding the experimental methods, the automatic data processing may be done either by using commercial software, or by developing a customized code for particular applications.

\section{THEORETICAL BACKGROUND}

Experimental mechanics offers several technologies to investigate the structural phenomena. Among them, one can remind: strain gage technology, PhotoStress method, Video Image Correlation, and others.

Photo-stress method is employed for a full-field study of the strains as well as quantitative measurements of the strains.A strain-sensitive plastic coating is bonded to the structure to be investigated. The structure is loaded and meanwhile the coating is illuminated with polarized light. The plastic and the part are deflected in the same way, so they have the same strains. On the plastic can be noticed isochromatics, which are series of successive and contiguous different-colored bands. Each color of the fringe identifies in a unique way the fringe order, which is the strain level along that band. The zero-strain area of the coating may be identified by its black color in comparison with the colored fringes around it.

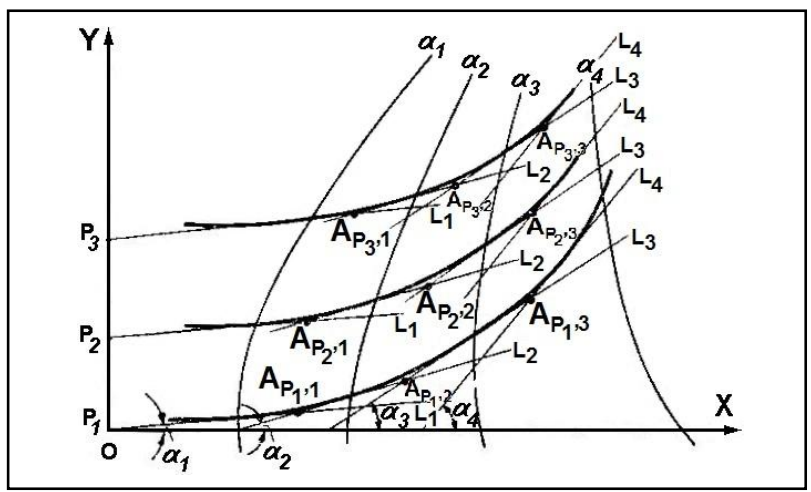

Fig. 2. Graphical method to draw the isostatics, [1]

Using a compensator there can be easily performed quantitative measurements of the strains.

Viewing the surface of the structure through a reflection polariscope, in all the points where the polarizer and the analyzer are parallel one to the other and both to the principal stresses, there can be noticed a black line on the coating, this line being called isoclinic. 
In each point of an isoclinic the directions of the principal strains are parallel to the direction of polarization, same in the analyzer and polarizer.

In order to perform quantitative measurements there must be measured the principal directions of the stresses.

The first step is to choose a reference line which might be the axis of symmetry or simply a vertical or horizontal line.With respect to this line, the assembly polarizer plus analyzer are rotated together until an isocline passes over the point where the directions must be measured.Having the set of isoclines, there can be drawn the isostatics, which are the principal stress trajectories. The next paragraph describes the graphical procedure employed to draw the isostatics using only the information provided by the isocline curves.

After the principal directions of the strains are known, there can be measured the principal strains.

For instance, the Babinet-Soleil compensator is placed in front of the investigated part, right over the measurement point with its optical axis oriented along the $\varepsilon_{2}$ strain (or $\sigma_{2}$ stress) and moving a prismatic plate it is applied a so called induced strain.

Other example is about the Tardy compensation method which has the following features:

- it is employed a circular light polariscope;

- there are used the dark field measurements;

- $\quad$ principal directions were previously established;

- the fringe numbers were correct measured.

If the polarizer and the analyzer are aligned with the principal stresses and the quarter-wave plates are places at $45^{\circ}$ with respect to the axis of the polarizer, the rotation of the analyzer with an $\alpha$ angle, will move the fringe in a position where the fringe number has the $\frac{\alpha}{180^{\circ}}$ value.

To conclude, an accurate solution regarding the location of the isostatics is paramount for the precision of the overall measurements.

\section{THE GRAPHIC PROCEDURE}

Let us consider the situation presented in figure 2 . Along the vertical axis let us consider a set of points $P_{i}$, $i=1, \ldots, n$ at equal distances one to the other.

Through point $P_{1}$ we consider line $L_{1}$ which has the $\alpha_{1}$ angle with respect to the horizontal axis, in accord with the $\alpha_{1}$ isocline. We denote by $A_{P_{1}, 1}$ the point located on $L_{1}$ at middistance between the $\alpha_{1}$ and $\alpha_{2}$ isoclines.

Through point $A_{P_{1}, 1}$ we consider line $L_{2}$ which has the $\alpha_{2}$ angle with respect to the horizontal axis, in accord with the $\alpha_{2}$ isocline. We denote by $A_{P_{1}, 2}$ the point located on $L_{2}$ at mid distance between the $\alpha_{2}$ and $\alpha_{3}$ isoclines.
The next stage is similar. Through point $A_{P_{1}, 2}$ we consider line $L_{3}$ which has the $\alpha_{3}$ angle with respect to the horizontal axis, in accord with the $\alpha_{3}$ isocline. We denote by $A_{P_{1}, 3}$ the point located on $L_{3}$ at mid distance between the $\alpha_{3}$ and $\alpha_{4}$ isoclines. The set of points designated by $A_{P_{i}, j}$ define the isostatic curve number ' $j^{\prime}$.

\section{ANALYTIC APPROACH}

The set of graphical operations previously described may be expressed in an analytic form. The input data consists of the points $P_{i}$, angles $\alpha_{i}$, and set of points which define the isoclines, $I_{\alpha_{i}, j}$ also denoted $I_{i, j}$.

The isoclines may be approximated either as linear functions, or as spline functions. In order to have a flexible application it will be employed a procedural modeling. Let us consider a segment of a line defined by the points $\left(x_{0}, y_{0}\right)$ and $\left(x_{1}, y_{1}\right)$, the resulting equation of the line being $\begin{aligned} \frac{y-y_{0}}{y_{1}-y_{0}}=\frac{x-x_{0}}{x_{1}-x_{0}} & \text { expressed as } \\ y & =\underbrace{\left(\frac{y_{1}-y_{0}}{x_{1}-x_{0}}\right)}_{m} \cdot x+\underbrace{\frac{\left(x_{1} \cdot y_{0}-x_{0} \cdot y_{1}\right)}{\left(x_{1}-x_{0}\right)}}_{n}\end{aligned}$

or

$$
y=m \cdot x+n
$$

One can notice the condition $x_{1} \neq x_{0}$, this means that the line should not be vertical and the two points must be distinct one to the other.

The intersection between the lines

$$
\left\{\begin{array}{l}
y=m_{1} \cdot x+n_{1} \\
y=m_{2} \cdot x+n_{2}
\end{array}\right.
$$

produces an intersection point whose coordinates are:

$$
x_{I}=-\frac{n_{1}-n_{2}}{m_{1}-m_{2}}, y_{I}=\frac{m_{1} \cdot n_{2}-m_{2} \cdot n_{1}}{m_{1}-m_{2}}
$$

if the two lines are not parallel, this means $m_{1} \neq m_{2}$. This point is considered valid only if it belongs to the segment, so its coordinates must be in the range defined by the coordinated of the point which define the segment. If we consider that the segment is defined by the points $\left(x_{1}^{S_{1}}, y_{1}^{S_{1}}\right)$ and $\left(x_{2}^{S_{1}}, y_{2}^{S_{1}}\right)$, where $x_{1}^{S_{1}}<x_{2}^{S_{1}}$, the according condition is $x_{1}^{S_{1}} \leq x_{I} \leq x_{2}^{S_{1}}$. The other condition may be written for $S_{1} \rightarrow S_{2}$, that is $x_{1}^{S_{2}} \leq x_{I} \leq x_{2}^{S_{2}}$.

Let us consider that the isoclines are approximated using spline curves. Once the points $I_{i, j}$ are provided there are computed the coefficients of the spline function for the current isocline ' $i$ ' and the current interval ' $j$ ', where $j=1, \ldots, N_{i}: C_{i, j}^{(0)}, \quad C_{i, j}^{(1)}, \quad C_{i, j}^{(2)}, \quad C_{i, j}^{(3)}$. The conditions of continuity for the function and the first derivative in each mid node and the boundary conditions 
at the both ends of the isoclinic curves lead to a system of equations having the previous $C_{i, j}^{(m)}, m=0,3$ coefficients as unknowns. Once solved, we can analytically express an isoclinic curve as a spline curve defined as a set of $3^{\text {rd }}$ order polynomial functions. It can be proven that higher orders are not necessary. For the ' $i$ ' isocline and the ' $j$ ' interval, the expression of the according spline function is:

$$
\begin{aligned}
& y-y_{I_{i, j}}= \\
& =C_{i, j}^{(3)} \cdot\left(x-x_{I_{i, j}}\right)^{3}+C_{i, j}^{(2)} \cdot\left(x-x_{I_{i, j}}\right)^{2}+C_{i, j}^{(1)} \cdot\left(x-x_{I_{i, j}}\right)+C_{i, j}^{(0)}
\end{aligned}
$$

For a given ' $i$ ' we define the function

$$
\begin{aligned}
& g_{i, j}(x)=C_{i, j}^{(3)} \cdot\left(x-x_{I_{i, j}}\right)^{3}+C_{i, j}^{(2)} \cdot\left(x-x_{I_{i, j}}\right)^{2}+C_{i, j}^{(1)} \cdot\left(x-x_{I_{i, j}}\right)+(6) \\
& +C_{i, j}^{(0)}+y_{I_{i, j}}
\end{aligned}
$$

If we want to solve the intersection problem between two curves which are approximated using spline functions, we define the function

$$
h(x)=g_{i=i_{1}, j}(x)-g_{i=i_{2}, k}(x)
$$

where $j=1, \ldots, N_{i=i_{1}}, k=1, \ldots, N_{i=i_{2}}$, and we have to solve the equation

$$
h(x)=0
$$

The solving methods to be used may be either Tartaglia (Cardano) 'exact' method, or a numerical method, such as the bisection method. The solution $x_{I}$, $y_{I}$ must satisfy the conditions: $x_{i=i_{1}, j} \leq x_{I} \leq x_{i=i_{1}, j+1}$ and $x_{i=i_{2}, k} \leq x_{I} \leq x_{i=i_{2}, k+1}$ for the current ' $j$ ' and ' $k$ ' for which condition (8) is satisfied.

If one of the geometrical entities is approximated as a linear function we can use equation (6) with the replacements:

$$
C_{i, j}^{(3)}=0 ; C_{i, j}^{(2)}=0 ; C_{i, j}^{(1)}=m_{i, j} ; C_{i, j}^{(0)}=n_{i, j} ;
$$

the resulting expression having the same general form as (6), an advantage for the programming stage of the project. However, in order to have a clear formulation of the problem, we use the symbol

$$
f_{i, j}(x)=m_{i, j}^{(1)} \cdot\left(x-x_{I_{i, j}}\right)+n_{i, j}^{(0)}+y_{I_{i, j}}
$$

The new function to be defined is similar with (7)

and in this case we can express it as:

$$
h(x)=f_{i=i_{1}, j}(x)-g_{i=i_{2}, k}(x)
$$

the according equation being (8). same.

The methods to solve the previous equation are the

Theresults of these calculi are the coordinates of the $A_{P_{i}, j}$ intersection points, denoted $X_{A_{P_{i}, j}}, Y_{A_{P_{i}, j}}$ which may be grouped with respect to ' $j$ 'which counts the interval between two successive isoclines. Being grouped in such way, there can be generated the analytical approximation for the isostatics as linear functions or as spline functions.
The symbols employed in the aforementioned presentation were chosen in such a way that they may be easily used as variables in the computer code to be developed.

\section{ACCURACY OF THE METHOD}

The accuracy of the method depends on a set of factors, such as:

- $\quad$ accuracy of the initial dataaset which approximates the isoclines;problems might appear for areas with great stress concentrators where many fringes can appear;

- automatic processing of the images representing the isoclines which can create the input data for this algorithm might be a solution for a large amount of data, but accuracy is still a problem to be studied, because the isoclines may be found as narrow bands or wide bands which must be approximated either automatically, or by a skilled experimental technician or analyst;

- precision of the mechanisms of the polariscope employed to generate the isoclines;

- a larger number of isoclines to be taken into consideration might increase the accuracy of the isostatics, and also the overall volume of calculi;

- the accuracy of the numerical methods employed to process the data, the datatypes, the desired attribute in the accuracy vs. speed paradigm, etc.

The most important input data consist of the set of isoclines. In order to have an accurate pattern of isoclines there must be taken into consideration the following important properties:

- isoclines do not intersect one to the other, except of the isotropic point defined as the points where $\sigma_{1}-\sigma_{2}=0$, in these point all the directions being principal directions;

- the $\alpha_{i}$ isocline is intersecting an unloaded contour in a point where the tangent has the same $\alpha_{i}$ angle, with respect to the horizontal direction;

- if the structure has a region of its contour unloaded and straight, this is an isocline because it is a line along which the principal stresses have the same direction;

- if the investigated part has an axis of symmetry, this is actually an isocline of a given angle;

- if the structure has some isotropic points, all the isoclines pass through these points;

- isoclines of all angles pass through the points where the concentrated loads are applied;

- in every point there are two principal directions which are perpendicular one to the other; it results that an isocline of $\alpha_{i}$ parameter is identical with the isocline of $\alpha_{i}+\frac{\pi}{2}$ parameter.

To conclude, even the algorithm has a low complexity, the accuracy must be checked, double checked and over checked using several test cases in order to find all the possible sources of errors and to keep the errors under control. 


\section{APPLICATION}

One can notice the technical progress of the instruments in experimental mechanics, including the polariscope systems, which offer today facile operation and accurate results. For instance, in the past 25 years the most popular instrument in this field was the 030 Series Modular Reflection Polariscope System from Vishay which offers a wide range of measurement capabilities. Several features were automatized in order to have more facilities, but there was no new module which can offer automatic drawing of the isostatics. However, it was developed a new innovative polariscope for accurate static strain measurements, the Model 040 Direct Digital Display Reflection Polariscope. It has several built-in features for direct display of the measured stresses and strains in static conditions.

The automatic data processing algorithm presented in the paper may be used to update the performances of the actual polariscopes employed in both education and research activities.

\section{CONCLUSION}

The limits of the study are presented in the previous section regarding the accuracy of the method, the most important source of errors being identified as the input initial pattern of isoclines which can be both wide bands and narrow bands.

The algorithm is simple, stable, accurate and it can be easily implemented and used. The basic ideas previously presented can be used in many other cases when graphs (like the results of the experimental tests) must be used for automatic data processing (design). Extension from 2D graphs to 3D surfaces may be easily done.

The use of this algorithm for the automatic data processing can offer a rapid overview regarding the stress distribution in a structure.

For a high degree of automation and generality of the data processing, the effectiveness of the technology is increased and it can offer full-field quantitative information. Moreover, having a large volume of accurate experimental results, they can be used for hybrid models. In this way the technology can be updated and it can offer competitive results with respect to the actual modern technologies.

It is advisable to proof the algorithm and its implementation using many case-studies in order to gain a high degree of confidence in its results. Case studies may be also used for educational purposes, [6], [7], [8].

\section{ACKNOWLEDGEMENTS}

Several of the ideas presented in the paper are the result of the models developed in the framework of the scientific research study 'Development of computer assisted marine structures', Emil Oanta, Cornel Panait, Ghiorghe Batrinca, Alexandru Pescaru, Alexandra Nita, Feiza Memet, which is a component of the RoNoMar project, 2010, sponsor of this paper.

Ideas regarding the computer based instruments in applied elasticity are the result of the models developed in the framework of the MIEC2010 bilateral Ro-Md research project, Oanta, E., Panait, C., Lepadatu, L., Tamas, R., Constantinescu, M., Odagescu, I., Tamas, I., Batrinca, G., Nistor, C., Marina, V., Iliadi, G., Sontea, V., Marina, V., Balan, V. (2010-2012), "Mathematical Models for Inter-Domain Approaches with Applications in Engineering and Economy", MIEC2010 - Bilateral Romania-Moldavia Scientific Research Project, under the supervision of the National Authority for Scientific Research (ANCS), Romania, that is the follow-up of the ID1223 scientific research project: Oanta, E., Panait, C., Nicolescu, B., Dinu, S., Pescaru, A., Nita, A., Gavrila, G., (2007-2010), "Computer Aided Advanced Studies in Applied Elasticity from an Interdisciplinary Perspective", under the supervision of the National University Research Council (CNCSIS), Romania.

\section{REFERENCES}

[1] Theocaris P. S.; Atanasiu C.; Boleantu L.; Buga M.; Burada C.; Constantinescu I.; Iliescu N.; Mocanu D. R.; Pastrav I.\& Teodoru M. (1976), Experimental stress analysis - Theoretical bases of the stress and strain measurement methods and practical indications, Vol I and II, Technical Publishing House, Bucharest, 1976

[2] Oanta, E.; Barhalescu, M. \& Sabau, A., (2011). Management of Change Based on Creative Inter-Domain Syntheses, Proceedings of the $7^{\text {th }}$ International Conference on Management of Technological Changes, September $1^{\text {st }}-3^{\text {rd }}, \quad 2011$, Alexandroupolis, Greece, Vol II, ISBN (Vol. II) 978-960-994863-0, ISBN 978-960-99486-1-6, Costache Rusu (Ed.), pp. 589-592, Democritus University of Thrace, Alexandroupolis, Greece, 2011

[3] Oanta, E.; Panait, C.; Batrinca, G. \& Pescaru, A., (2011). Basic Concepts to Design the Software Application of a Computer Based Mechanical Engineering Model, Annals of DAAAM for 2011 \& Proceedings of the $22^{\text {nd }}$ International DAAAM Symposium, ISBN 978-3-901509-83-4, ISSN 1726-9679, pp 0505-0506, Branko Katalinic (Ed.), Published by DAAAM International, Vienna, Austria 2011

[4] Oanta, E.; Panait, C.; Batrinca, G. \& Pescaru, A., (2011). Computer Based Educational Model of the Bent Hull in the Context of the Maritime Education, Annals of DAAAM for 2011 \& Proceedings of the $22^{\text {nd }}$ International DAAAM Symposium, ISBN 978-3-901509-83-4, ISSN 1726-9679, pp 0503-0504, Branko Katalinic (Ed.), Published by DAAAM International, Vienna, Austria 2011

[5] Oanta, E.; Panait, C.; Marina, V.; Marina, V.; Lepadatu, L.; Constantinescu, E.; Barhalescu, M. L.; Sabau, A. \& Dumitrache, C. L., (2011). Mathematical Composite Models, a Path to Solve Research Complex Problems, Annals of DAAAM for 2011 \& Proceedings of the $22^{\text {nd }}$ International DAAAM Symposium, ISBN 978-3-901509-83-4, ISSN 1726-9679, pp 0501-0502, Branko Katalinic (Ed.), Published by DAAAM International, Vienna, Austria 2011

[6] Oanta, E. (2012); Solved problems of Strength of Materials with Applications in Marine Engineering - Examples of examination subjects, Nautica Publishing House, ISBN 978-606-8105-65-9, Constanta Romania

[7] Sabau, A.; Oanta, E. \& Barhalescu, M., (2011). Impact Of The Use Of The Modern Methods In The Training Of Marine Engineer Cadets, Proceedings of the $7^{\text {th }}$ International Conference on Management of Technological Changes, September $1^{\text {st }}-3^{\text {rd }}$, 2011, Alexandroupolis, Greece, Vol II, ISBN (Vol. II) 978-96099486-3-0, ISBN 978-960-99486-1-6, Costache Rusu (Ed.), pp. 421-424, Democritus University of Thrace, Alexandroupolis, Greece, 2011

[8] Sabau, A.; Barhalescu, M. \& Oanta, E., (2011). Simulation Programs in Teaching Activity, Proceedings of the $7^{\text {th }}$ International Conference on Management of Technological Changes, September $1^{\text {st }}-3^{\text {rd }}$, 2011, Alexandroupolis, Greece, Vol II, ISBN (Vol. II) 978-960-99486-3-0, ISBN 978-960-99486-1-6, Costache Rusu (Ed.), pp. 425-428, Democritus University of Thrace, Alexandroupolis, Greece, 2011 